\title{
Nuclear Hepatology: A Textbook of Hepatobiliary Diseases
}

\author{
G.T. Krishnamurthy and S. Krishnamurthy
}

New York, NY: Springer, 2009, 368 pages, $\$ 199$

This second-edition textbook on nuclear hepatology and hepatobiliary diseases is well written, handy, and supplemented with good illustrations and images that effectively explain hepatic physiology and pathology starting at the cellular microstructure level and proceeding to the visualized nuclear scan findings. The meticulous attention paid to minute detail throughout the book is a testimony to the authors' sincere and intense interest in nuclear hepatology. The book covers all aspects of nuclear hepatology from embryology, morphology, structure, anatomy, and physiology, to effective descriptions of the nuclear scan findings as a reflection of the functioning of the hepatobiliary system in health and disease. This comprehensive book makes us pause, analyze, and reason out the physiology or pathology behind the normal or abnormal hepatic scan findings in a given patient. Each chapter has an adequate number of references and is supplemented with good-quality pictorial illustrations, tables, graphs, diagrams, and images to explain the physiology and pathology seen on the nuclear scans. Illustrative examples are given of gallbladder emptying resulting from the interaction of cholecystokinin with its receptors on the gallbladder in normal patterns and in chronic cholecystitis or bile flow patterns in cystic ducts and sphincter-of-Oddi dysfunction. These examples make it easier to understand and identify the hepatoiminodiacetic acid (HIDA) scan patterns in these different conditions. The book walks us through the liver disease process starting with the initial reversible stage of prolongation of the hepatic transit time to disruption of the basolateral border, cell rupture, and final cell death, thereby helping us to understand the effects of the disease process on the nuclear scan.

Chapter 1 covers the morphology and microstructure of the hepatobiliary system from embryology and segmental anatomy to vasculature, lymphatics, nerves, the common bile duct, and the gallbladder. The microstructure and function of the different hepatic cells and the role of the gallbladder and sphincter of Oddi in HIDA imaging are well described. The illustrations and images in this chapter make it easier to understand these topics.

Chapter 2 comprehensively covers liver function, including the basolateral and canalicular domains of the hepatocytes, the sodium-dependent pathways, the role of the organic anion-transporting polypeptide in the sodium-inde-

COPYRIGHT @ 2010 by the Society of Nuclear Medicine, Inc. DOI: 10.2967/jnumed.110.081034 pendent pathways, the basolateral transporters, the nuclear receptors, apoptosis, and protein secretion. Again, the many detailed, clear illustrations make this complex chapter easier to understand. Bile entry, storage, concentration, and emptying of the gallbladder give us the physiologic rationale for HIDA scanning. Chapter 3 covers the different hepatic imaging agents, from the infrequently used ${ }^{99 \mathrm{~m}} \mathrm{Tc}$-sulfur colloid to the routinely used HIDA agents. Starting with their uptake in the hepatocyte, the authors describe their hepatic transit, secretion into the bile canaliculi, storage in the gallbladder, and final biliary excretion into the duodenum, including the effect of high serum bilirubin levels on the various iminodiacetic acid agents. Other infrequently used hepatic imaging agents are briefly covered, such as ${ }^{99 \mathrm{~m}} \mathrm{Tc}$-labeled red blood cells for hemangioma imaging, ${ }^{111} \mathrm{In}-$ or ${ }^{99 \mathrm{~m}} \mathrm{Tc}-$ labeled white blood cells for infection imaging, ${ }^{111}$ In-pentetriotide (OctreoScan; Covidien) for neuroendocrine tumors, ${ }^{67} \mathrm{Ga}$ imaging in hepatocellular carcinoma, and ${ }^{18} \mathrm{~F}$-FDG.

Chapter 5 is on hepatobiliary imaging and quantification of hepatobiliary function and describes normal and abnormal HIDA findings and the role of cholecystokinin in gallbladder emptying and ejection fraction. Other topics that round out this chapter include evaluation of hepatopulmonary syndrome using ${ }^{99 \mathrm{~m}} \mathrm{Tc}$-macroaggregated albumin, quantification of duodenogastric reflux, evaluation of arterial and portal-venous blood flow, and the effect of opioids on the sphincter. This chapter introduces us to the newer agent ${ }^{99 \mathrm{~m}} \mathrm{Tc}$-galactosyl human serum albumin for imaging and quantification of the hepatocyte asialoglycoprotein receptors-useful in predicting liver function after resection. Chapter 6 is on the effects of cholecystokinin and morphine on the gallbladder and sphincter of Oddi and the roles of these agents in HIDA scanning. Chapters 7-10 are clinically relevant, explaining HIDA scanning in intrahepatic and extrahepatic cholestasis, gallbladder disease, and bile duct disease. Chapter 11, on pediatric nuclear hepatology, explains the usefulness of phenobarbital-augmented HIDA scanning in biliary atresia, giving helpful hints to make it easier to differentiate neonatal hepatitis from biliary atresia on HIDA scans. Chapter 12 is on the role of ${ }^{18} \mathrm{~F}$-FDG PET in primary and metastatic liver disease, and chapter 13 covers the role of HIDA scanning in pre- and post-liver transplant evaluation.

It is obvious from this meticulously written textbook that the Drs. Krishnamurthy have devoted extensive time and 
research to the field of nuclear hepatology, a topic near and dear to their hearts. The book reflects their painstaking attention to details and helps us to better understand how the HIDA scan accurately shows the physiology and pathologic processes occurring at the hepatobiliary structural level.

The book has a few minor editorial issues. On page 144, the gallbladder ejection fraction is mistakenly referred to as "ejection period." On page 62, under "Gallbladder Storage," the time of $180 \mathrm{~min}$ is incorrectly translated to $6 \mathrm{~h}$ rather than $3 \mathrm{~h}$. On page 293, Figure 9.4.3, the right-hand images are inaccurately labeled as $2 \mathrm{~d}$ after cholecystectomy rather than the $12 \mathrm{~d}$ described in the accompanying figure legend.

A helpful addition to round out the book would be a section on the use of ${ }^{99 \mathrm{~m}}$ Tc-macroaggregated albumin scanning to quantify lung uptake and identify extrahepatic abdominal activity before ${ }^{90} \mathrm{Y}$ therapy for liver malignancy or metastasis, since this therapy is increasingly being used in many hospitals and medical centers today.
Overall, this is a well-written, informative, useful, handy reference book filled with detailed information, excellent illustrations, and good nuclear hepatology and nuclear hepatic images. The book expands and increases our basic understanding of the microstructure, physiology, and pathology of liver function in health and disease and helps us to understand how these conditions translate to the findings seen on hepatic scans. I highly recommend the book to nuclear and radiology departmental and hospital libraries. It will also be a valuable addition to the nuclear physician's personal medical library, serving as an excellent reference resource for radiologists, nuclear physicians, residents, and medical students confronted with routine or unusual hepatic scan findings.

Usha A. Joseph

Department of Radiology/Nuclear Medicine

UT Medical School at Houston

Houston, TX 77030 